\title{
Proteomic Profiling and Bioinformatics Analyses of Low-Level Laser-Treated Hypoxic Astrocytes
}

\author{
Yi-Jen Chen ${ }^{1,2}$, Chia-Hsin Chen ${ }^{1,3}$, Yu-Chang Tyan ${ }^{4}$, Wan-Jou Chen ${ }^{3}$ \\ ${ }^{1}$ Department of Physical Medicine and Rehabilitation, Kaohsiung Medical University Hospital, Taiwan \\ ${ }^{2}$ Graduate Institute of Clinical Medicine, College of Medicine, Kaohsiung Medical University, Taiwan \\ ${ }^{3}$ Department of Physical Medicine and Rehabilitation, School of Medicine, Kaohsiung Medical University, Taiwan \\ ${ }^{4}$ Department of Medical Imaging and Radiological Sciences, Kaohsiung Medical University, Taiwan
}

Background and Aims: The application of low-level laser therapy (LLLT) in brain ischemia provides clinical significance in stroke management. The aim of this study was to explore molecular signatures underlying the neuroprotective effect of LLLT in hypoxic astrocytes.

\section{Methods:}

- Mouse astrocytes under normoxic or hypoxic environment

- Confirm hypoxia-inducible factors expression

- MTT assay to assess cell viability of astrocytes after hypoxia

- LLLT at $4 \mathrm{~J} / \mathrm{cm}^{2}$ for 2 days

- Cell lysates for proteomic profiling using LC-MS/MS analyses

- Differentially expressed (DE) proteins between normoxic, hypoxic and LLLT-treated astrocytes analyzed

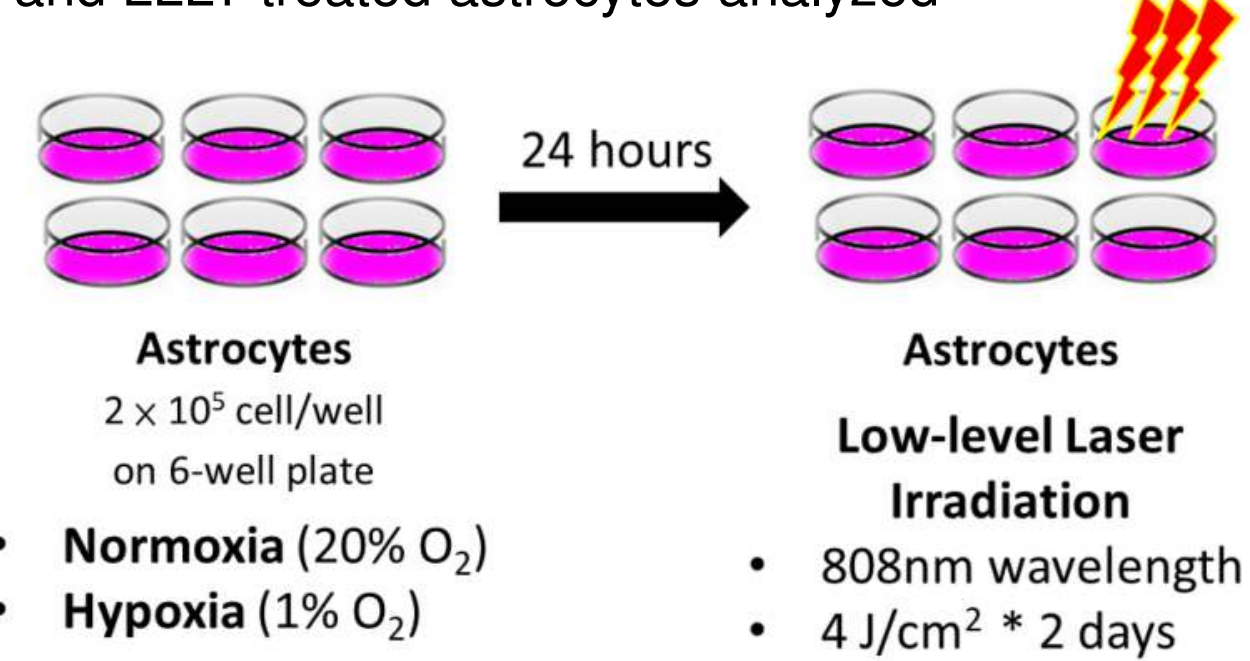

Result 1: Confirmation of hypoxic induction of astrocytes

- No significant difference in cell viability by MTT assay

- Increased expression of HIF-2a in hypoxic-treated astrocytes
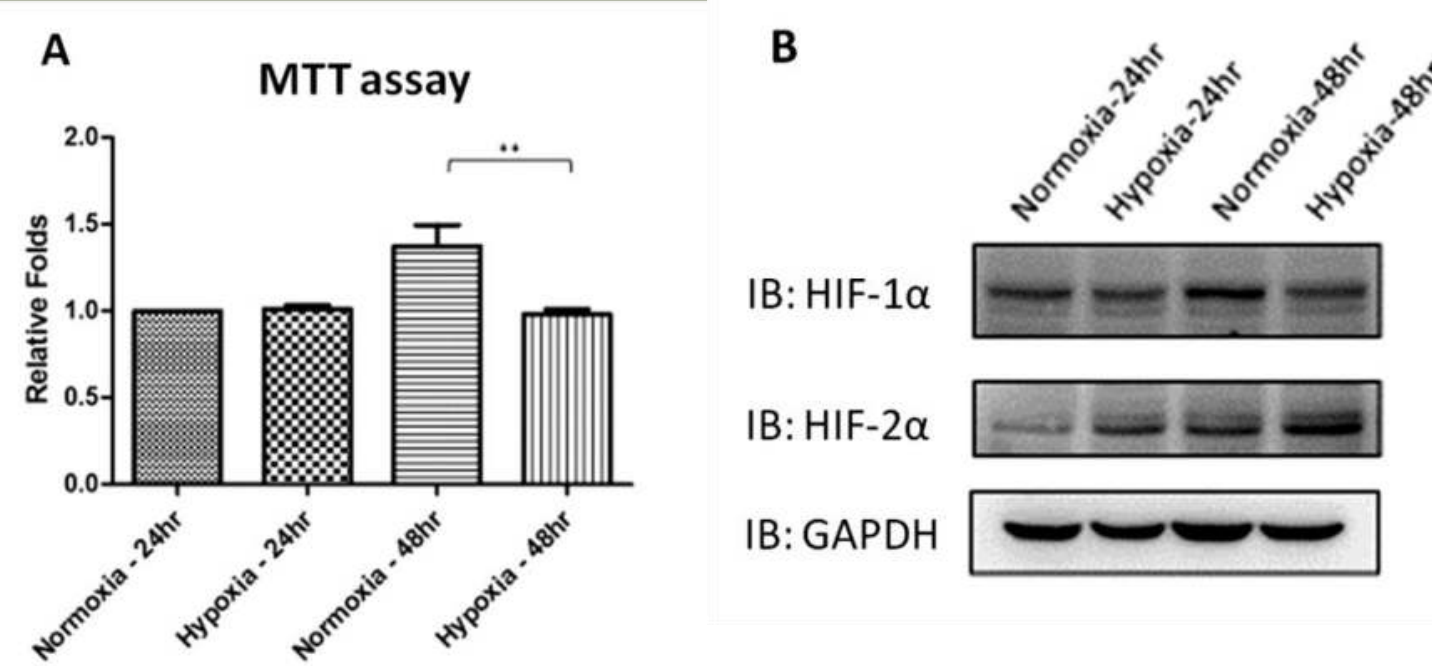

Result 2: Bioinformatics analyses of DE proteins in hypoxic-treated astrocytes

- 25 DE proteins identified in hypoxic astrocytes

- Involved in glucose metabolism

- ERK1/2 as central connecting hub

- Increased HS90B, LDHA and THIO involved in HIF signaling and NRF2-mediated oxidative stress response

Table 1. Top canonical pathways

\begin{tabular}{lcc}
\hline Canonical pathways & Molecules & p-value \\
\hline Gycolysis & PGAM1,ALDOA,ENO1 & $2.96 \times 10^{-6}$ \\
Gluconeogenesis & PGAM1,ALDOA,ENO1 & $2.96 \times 10^{-6}$ \\
EIF2 Signaling & RPL21,EIF3B,EIF4A3 & $1.76 \times 10^{-3}$ \\
Hypoxia Signaling in the Cardiovascular System & LDHA,HSP90AB1 & $3.05 \times 10^{-3}$ \\
Diphthamide Biosynthesis & EEF2 & $3.29 \times 10^{-3}$ \\
\hline
\end{tabular}

Table 2. KEGG pathways by DAVID database analysis

\begin{tabular}{lccc}
\hline KEGG pathway & P value & Molecules & $\begin{array}{c}\text { Fold } \\
\text { Enrichment }\end{array}$ \\
\hline Glycolysis / Gluconeogenesis & $3.73^{*} 10^{-4}$ & ALDOA, ENOA, LDHA, PGAM1 & 25.99 \\
Biosynthesis of antibiotics & 0.011 & ALDOA, ENOA, LDHA, PGAM1 & 8.02 \\
Biosynthesis of amino acids & 0.012 & ALDOA, ENOA, PGAM1 & 16.50 \\
Glucagon signaling pathway & 0.020 & LDHA, CALM, PGAM1 & 12.87 \\
Carbon metabolism & 0.026 & ALDOA, ENOA, PGAM1 & 11.09 \\
Spliceosome & 0.034 & ROA3, HNRPU, IF4A3 & 9.67 \\
\hline
\end{tabular}

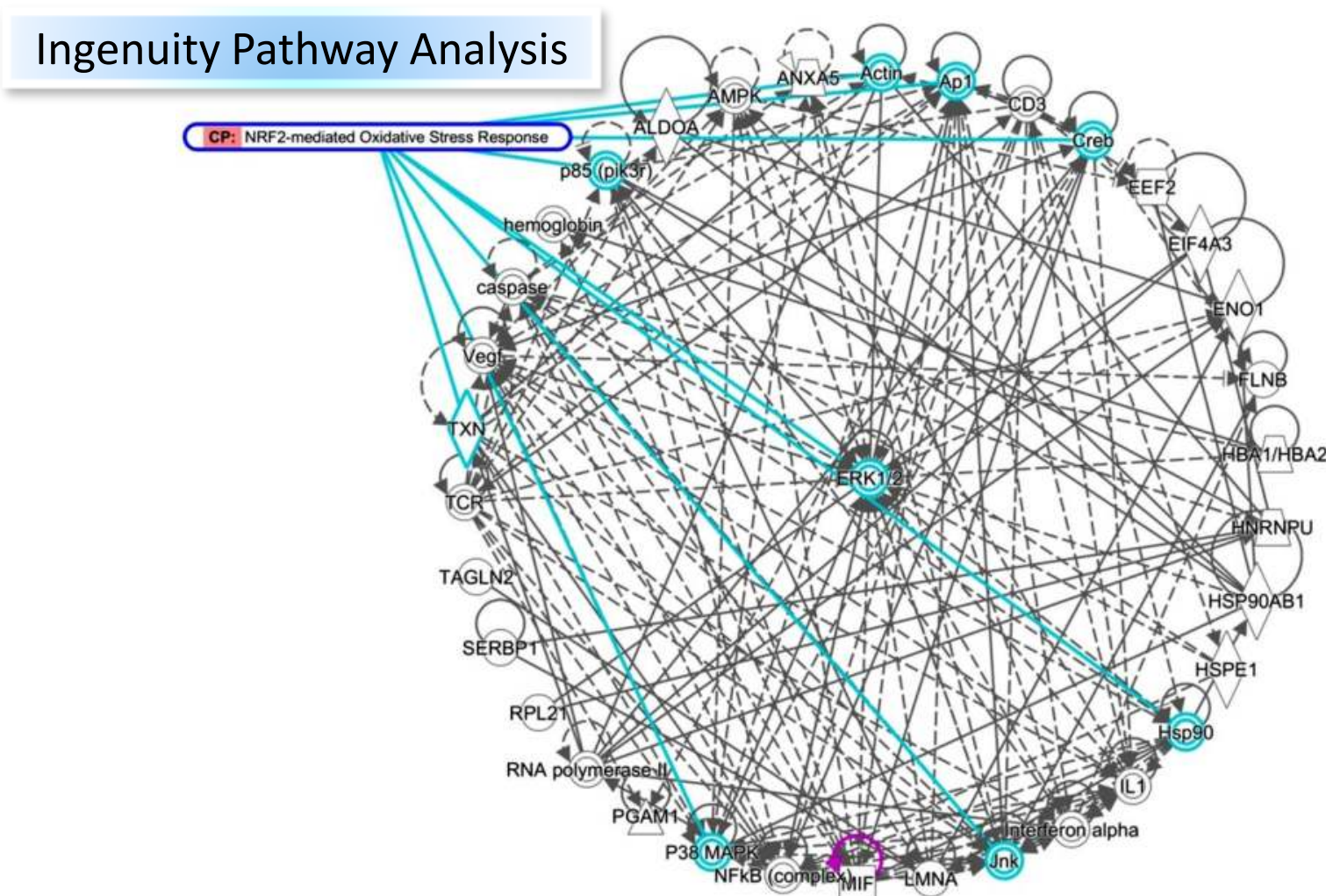

Result 3: Bioinformatics analyses of DE proteins in lasertreated hypoxic astrocytes

- 17 DE proteins identified in laser-treated hypoxic astrocytes

- 6 proteins involved in post-translational protein regulation through acetylation

- Filamin (FLNA) interact with HS90B, potentially facilitated LLLT-treated hypoxic astrocyte migration

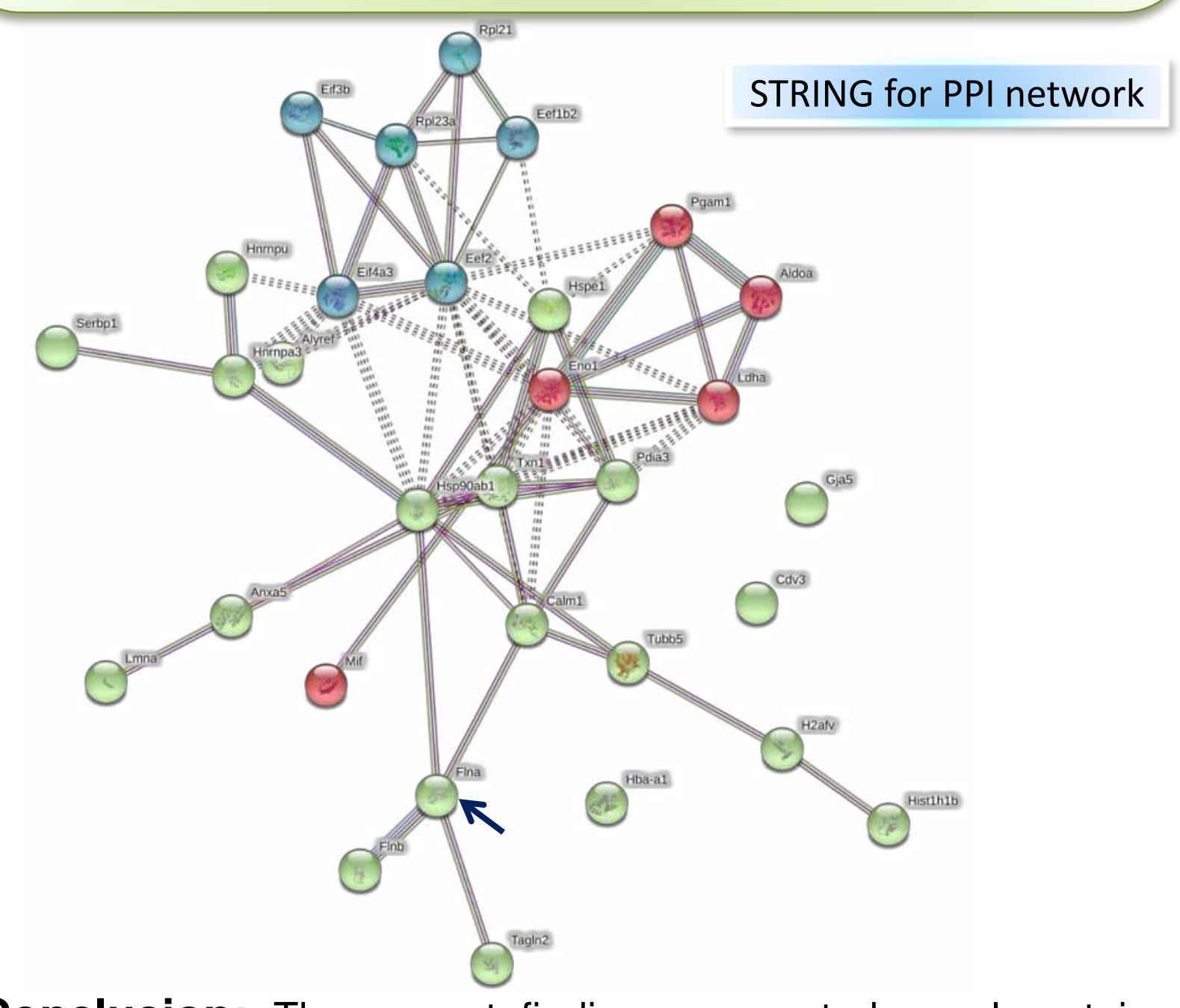

Conclusion: The current findings suggested novel proteins involved in the potential mechanisms of neuroprotective effects exerted by LLLT in brain ischemia. Further in vitro validation to confirm the hypothesis is necessary.

\section{References:}

1. Yang MH, et al. Mass spectrometry in clinical diagnosis: a preliminary application in tumor cellular proteomics for biomarker discovery. J Anal Bioanal Tech. 2014;:S2:009.
2. Thomas S, Bonchev D. A survey of current software for network analysis in molecular biology. Hum Genomics. 2010;4:353-60.

3. Huang da W, et al. Systematic and integrative analysis of large gene lists using DAVID bioinformatics resources. Nat Protoc. 2009;4:44-57.

4. Szklarczyk D, et al. String v10: Protein-protein interaction networks, integrated over the tree of life. Nucleic Acids Res. 2015;43:D447-452. 